Research Paper

\title{
Risk factors for progression to castration-resistant prostate cancer in metastatic prostate cancer patients
}

\author{
Ting-Ting Lin*, Ye-Hui Chen*, Yu-Peng Wu*, Shao-Zhan Chen*, Xiao-Dong Li, Yun-Zhi Lin, Shao-Hao \\ Chen, Qing-Shui Zheng, Yong Wei, Ning Xu ${ }^{\bowtie}, X_{\text {ue-Yi Xue }}{ }^{\bowtie}$ \\ Departments of Urology, the First Affiliated Hospital of Fujian Medical University, 20 Chazhong Road, Fuzhou 350005, China \\ *These authors contributed equally to this work. \\ $\square$ Corresponding authors: Xue-Yi Xue and Ning Xu, Department of Urology, First Affiliated Hospital of Fujian Medical University. 20 Chazhong Road, Fuzhou \\ 350005, China. Tel.: +86-059187981687; E-mail address: xuexueyi@fjmu.edu.cn (Xue-Yi Xue); drxun@fjmu.edu.cn (Ning Xu) \\ (1) The author(s). This is an open access article distributed under the terms of the Creative Commons Attribution License (https://creativecommons.org/licenses/by/4.0/). \\ See http://ivyspring.com/terms for full terms and conditions.
}

Received: 2018.10.16; Accepted: 2019.06.22; Published: 2019.09.07

\begin{abstract}
Purpose: To investigate the risk factors for progression to castration-resistant prostate cancer (CRPC) in metastatic prostate cancer ( $\mathrm{mPCa}$ ) patients who underwent androgen deprivation therapy (ADT).

Methods: We analyzed 216 patients with mPCa who underwent ADT between January 2006 and December 2015 at the First Affiliated Hospital of Fujian Medical University. Univariate and multivariate Cox regression analysis were used to explore the risk factors for progression to CRPC. Kaplan-Meier analysis and log-rank test were used to evaluate the difference in progression-free survival (PFS).

Results: A total of 121 (56.0\%) patients who underwent ADT showed progression to CRPC. Multivariate Cox regression analysis demonstrated that Gleason grade group, prostate-specific antigen nadir (nPSA), and time to PSA nadir (TTN) were risk factors for progression to CRPC in mPCa patients. Kaplan-Meier analysis demonstrated that patients in Gleason grade group $\geq 3, n P S A>0.2 \mathrm{ng} / \mathrm{ml}$ and TTN $<6$ months had shorter PFS.

Conclusion: This study demonstrated that Gleason grade group, nPSA and TTN were risk factors for progression to CRPC. Patients with higher Gleason grade group, higher nPSA and shorter TTN have shorter PFS and higher risk of progression to CRPC after ADT.
\end{abstract}

Key words: metastatic prostate cancer, androgen deprivation therapy, castration-resistant prostate cancer, progression-free survival, risk factors

\section{Introduction}

Recently, prostate cancer (PCa) has become one of the most common malignant tumors in western countries [1-3]. The incidence of PCa in China has increased in recent years [4]. Patients with bone or visceral metastasis can be diagnosed with metastatic PCa (mPCa) [5].

Patients with mPCa usually have no opportunity to undergo radical treatment. Instead, androgen deprivation therapy (ADT) works as the first-line treatment to prevent progression to castrationresistant PCa (CRPC)[6]. However, patients who undergo ADT inevitably show progression to CRPC [7]. Patients with CRPC have poor prognosis and unsatisfactory therapeutic effects.

Thus, it is important to identify the risk factors for rapid progression to CRPC in patients who initially respond to ADT. This study explored the risk factors for progression to CRPC in patients with mPCa who underwent ADT.

\section{Materials and Methods \\ Patients and data collection}

We retrospectively reviewed the clinical data of mPCa patients between January 2006 and December 2015. The regiments of patients received ADT in this study were luteinizing hormone-releasing hormone agonists (LHRH-A) accompanied with an antiandrogen until progression to CRPC or at the end of follow-up time. The inclusion criteria were as follows: patients diagnosed with pathologically confirmed PCa and have positive findings of bone or visceral 
metastasis. The exclusion criteria were as follows: patients with other cancer, treated with 5 a-reductase inhibitors within 6 months, received chemotherapy or radiotherapy during the follow-up time, or without whole course intervention of ADT were excluded in this study. Age, body mass index, prostate-specific antigen (PSA) baseline level, Gleason score and Gleason grade group were recorded in a database, as well as the level of alkaline phosphatase, lactate dehydrogenase and hemoglobin. Gleason score was subject to pathological diagnosis by prostate biopsy. Patients were divided into 5 groups according to the Gleason grade group system devised in 2014 by the International Society of Urological Pathology [8]: Grade group 1, Gleason score $\leq 6$; Grade group 2, Gleason score 3+4=7; Grade group 3, Gleason score 4+3=7; Grade group 4, Gleason score 8; and grade group 5, Gleason score $\geq 9$. Bone and visceral metastasis were diagnosed by bone emission computed tomography, magnetic resonance imaging of the pelvis, and computed tomography of the chest and abdomen. All patients received ADT regularly.

\section{Follow-up}

The duration of response to ADT in each patient was determined by the detection of serum PSA levels every 3 months. PSA nadir (nPSA) and time to PSA nadir (TTN) were recorded during follow-up. nPSA was classified as $\leq 0.2 \mathrm{ng} / \mathrm{ml}$ or $>0.2 \mathrm{ng} / \mathrm{ml}$, and TTN as $<6$ months or $\geq 6$ months, based on previously published studies [9-11]. The progression to CRPC was defined as castrate serum testosterone $<50 \mathrm{ng} / \mathrm{dl}$ or $1.7 \mathrm{nmol} / \mathrm{l}$, in addition to either biochemical progression ( 3 consecutive rises in PSA 1 week apart, resulting in 50\% increases over the nadir, with PSA $>2$ $\mathrm{ng} / \mathrm{ml}$ ), or radiological progression, based on the 2017 European Association of Urology guidelines [12, 13].

\section{Statistical analysis}

Statistical analysis was performed using SPSS version 22.0 (SPSS, Chicago, IL, USA). Survival curves were generated using the Kaplan-Meier method. Univariate and multivariate Cox regression analyses were performed to explore the risk factors associated with progression to CRPC. $\mathrm{P}<0.05$ was considered statistically significant.

\section{Results}

A total of 635 patients were diagnosed as metastatic prostate cancer in our center during the study period. A number of 419 patients were excluded in this study. 8 patients diagnosed with other cancers, 293 patients received 5a-reductase inhibitors or chemotherapy or radiotherapy, 11 patients refused to receive $\mathrm{ADT}$, and 107 patients without complete follow-up data were excluded from this study. Finally, we included 216 patients with mPCa (Table 1). Bone metastasis was found in $210(97.2 \%)$ cases, axial metastasis in $192(88.9 \%)$, limb bone metastasis in $66(30.6 \%)$, visceral metastasis in $46(21.3 \%)$, and lymph node metastasis in 198 (91.7\%).

Table 1. Characteristics of patients with metastatic prostate cancer underwent androgen deprivation.

\begin{tabular}{|c|c|c|}
\hline \multicolumn{2}{|l|}{ 我 } & $\mathrm{X} \pm \mathrm{S} / \mathrm{No}$ \\
\hline Age (year) & & $70.86 \pm 8.15(47-86)$ \\
\hline \multicolumn{2}{|l|}{ BMI $\left(\mathrm{Kg} / \mathrm{m}^{2}\right)$} & $22.29 \pm 3.15(16.10-30.40)$ \\
\hline \multicolumn{2}{|l|}{ Gleason score } & $8.06 \pm 1.22(4-10)$ \\
\hline \multicolumn{3}{|c|}{ Gleason grade group (\%) } \\
\hline Grade group1 & $\leq 6$ points & $29(13.4)$ \\
\hline Grade group2 & $3+4$ points & $37(17.1)$ \\
\hline Grade group3 & $4+3$ points & $23(10.6)$ \\
\hline Grade group4 & 8 points & $48(22.2)$ \\
\hline Grade group5 & $\geq 9$ points & $79(36.6)$ \\
\hline \multicolumn{3}{|c|}{ Lymph node metastasis (\%) } \\
\hline \multicolumn{2}{|l|}{ No } & $18(8.3)$ \\
\hline \multicolumn{2}{|l|}{ Yes } & 198 (91.7) \\
\hline \multicolumn{3}{|c|}{ Limb bone metastasis (\%) } \\
\hline \multicolumn{2}{|l|}{ No } & $150(69.4)$ \\
\hline \multicolumn{2}{|l|}{ Yes } & $66(30.6)$ \\
\hline \multicolumn{3}{|c|}{ Axial bone metastasis (\%) } \\
\hline \multicolumn{2}{|l|}{ No } & $24(11.1)$ \\
\hline \multicolumn{2}{|l|}{ Yes } & $192(88.9)$ \\
\hline \multicolumn{3}{|c|}{ Visceral metastasis (\%) } \\
\hline \multicolumn{2}{|l|}{ No } & $170(78.7)$ \\
\hline \multicolumn{2}{|l|}{ Yes } & $46(21.3)$ \\
\hline \multicolumn{2}{|c|}{ Alkaline phosphatase (U/L) } & $188.71 \pm 185.14$ \\
\hline \multicolumn{2}{|c|}{ Lactate dehydrogenase $(\mathrm{U} / \mathrm{L})$} & $223.94 \pm 185.17(66-1392)$ \\
\hline \multicolumn{2}{|c|}{ Hemoglobin $(\mathrm{g} / \mathrm{L})$} & $125.76 \pm 20.23(58-153)$ \\
\hline \multicolumn{3}{|c|}{ PSA baseline level (\%) } \\
\hline \multicolumn{2}{|l|}{$\leq 65 \mathrm{ng} / \mathrm{ml}$} & $168(77.8)$ \\
\hline \multicolumn{2}{|l|}{$>65 \mathrm{ng} / \mathrm{ml}$} & $48(22.2)$ \\
\hline \multicolumn{3}{|l|}{ nPSA (\%) } \\
\hline \multicolumn{2}{|l|}{$\leq 0.2 \mathrm{ng} / \mathrm{ml}$} & $82(38.0)$ \\
\hline \multicolumn{2}{|l|}{$>0.2 \mathrm{ng} / \mathrm{ml}$} & $134(62.0)$ \\
\hline \multicolumn{2}{|l|}{ TTN (\%) } & $8.10 \pm 6.99(2-42)$ \\
\hline \multicolumn{2}{|l|}{$<6$ months } & $115(53.2)$ \\
\hline \multicolumn{2}{|l|}{$\geq 6$ months } & $101(46.8)$ \\
\hline
\end{tabular}

Progression-free survival (PFS) is shown in (Figure 1). A total of 121(56.0\%) patients developed to CRPC. All patients included in this study were diagnosed to have progression to CRPC by PSA changes and a total of 82 patients were diagnosed to have progression to CRPC by imaging. The median follow-up time was 14.0 (3.0-88.0) months, and the median PFS was 14.7 (3.0-74.0) months. The median nPSA was $3.23(0-18.1) \mathrm{ng} / \mathrm{ml}$, and median TTN was $8.10(2-42)$ months.

Univariate Cox regression analysis showed that Gleason grade group, limb bone metastasis, visceral metastasis, alkaline phosphatase, PSA baseline level, nPSA, and TTN were significantly associated with progression to CRPC. Multivariate analysis revealed that patients in Gleason grade group 3 showed a 3.169-fold higher risk for progression to CRPC than those in group $1(\mathrm{P}=0.006)$. Furthermore, patients in 
Gleason grade group 4 showed a 4.335-fold higher risk for progression to CRPC than those in group 1 $(\mathrm{P}<0.001)$. Finally, patients in Gleason grade group 5 showed a 5.159-fold higher risk for progression to CRPC than those in group $1(\mathrm{P}<0.001)$. $\mathrm{P}$ for trend was calculated and showed that increasing Gleason grade group was significantly associated with higher risk of progression to CRPC ( $\mathrm{P}$ for trend <0.001). Multivariate Cox regression analysis also showed that $\mathrm{nPSA}>0.2 \mathrm{ng} / \mathrm{ml}$ (hazard ratio $2.665,95 \%$ confidence interval 1.495-4.750, $\mathrm{P}<0.001$ ) was associated with poor PFS when compared with nPSA $\leq 0.2 \mathrm{ng} / \mathrm{ml}$. However, TTN $\geq 6$ months (hazard ratio $0.262,95 \%$ CI 0.161-0.426) (Tables 2 and 3) was associated with better PFS when compared with TTN $<6$ months.

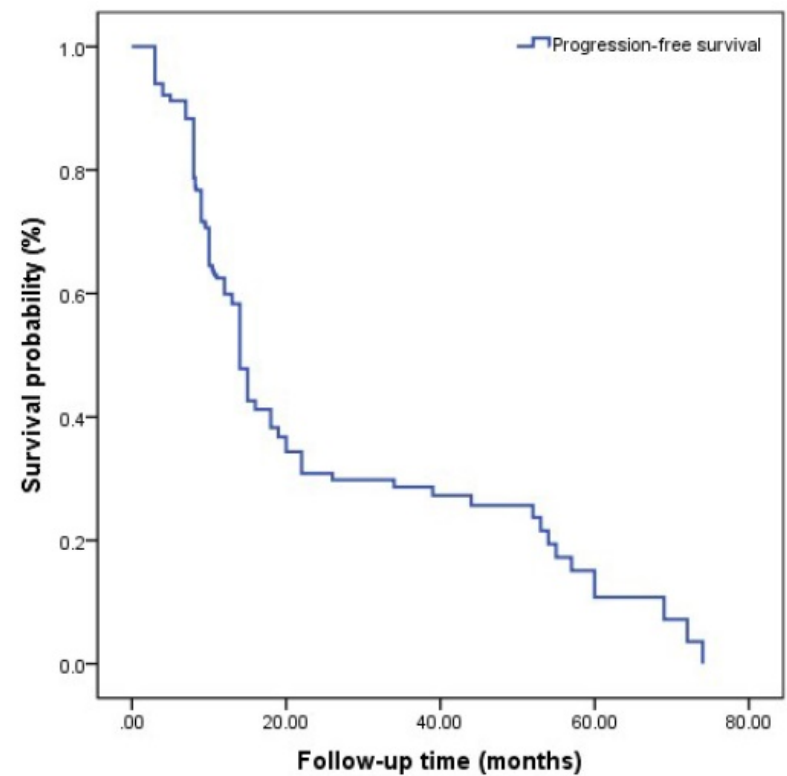

Figure 1. Kaplan-Meier analysis for progression-free survival in patients with advanced prostate cancer underwent androgen deprivation therapy.

The survival curves among different Gleason grade groups, nPSA, and TTN were generated (Figures 2-4). The results demonstrated that there were significant differences in PFS between patients with different Gleason grade group, nPSA and TTN (all $\mathrm{P}<0.001$ ). PFS was worse in patients with increasing Gleason grade group, higher nPSA and shorter TTN.

Table 2. Univariate analysis for progression to metastatic castration resistant prostate cancer.

\begin{tabular}{llll}
\hline & HR & 95\%CI & P Value \\
\hline Age & 1.006 & $0.986-1.025$ & 0.571 \\
BMI & 0.981 & $0.931-1.035$ & 0.487 \\
Gleason grade group & & & \\
Grade group1 $\leq$ 6 points & 1.000 & & \\
Grade group2 3+4 points & 1.493 & $0.562-3.963$ & $0.042^{*}$ \\
Grade group3 4+3 points & 2.680 & $1.226-5.857$ & $0.013^{*}$ \\
Grade group4 8 points & 8.722 & $4.043-18.818$ & $<0.001^{*}$ \\
\hline
\end{tabular}

\begin{tabular}{|c|c|c|c|}
\hline & HR & $95 \% \mathrm{CI}$ & P Value \\
\hline Grade group $5 \geq 9$ points & 13.181 & $6.218-27.942$ & $<0.001^{*}$ \\
\hline \multicolumn{4}{|l|}{$P$ for trend $<0.001^{*}$} \\
\hline \multicolumn{4}{|l|}{ Lymph node metastasis } \\
\hline No & 1.000 & & \\
\hline Yes & 1.022 & $0.926-1.496$ & 0.248 \\
\hline \multicolumn{4}{|l|}{ Limb bone metastasis } \\
\hline No & 1.000 & & \\
\hline Yes & 1.871 & $1.332-2.628$ & $<0.001^{*}$ \\
\hline \multicolumn{4}{|l|}{ Axial bone metastasis } \\
\hline No & 1.000 & & \\
\hline Yes & 0.847 & $0.502-1.428$ & 0.532 \\
\hline \multicolumn{4}{|l|}{ Visceral metastasis } \\
\hline No & 1.000 & & \\
\hline Yes & 0.625 & $0.406-0.960$ & $0.032^{*}$ \\
\hline Alkaline phosphatase & 1.001 & $1.000-1.002$ & $0.019^{*}$ \\
\hline Lactate dehydrogenase & 1.001 & $1.000-1.001$ & 0.116 \\
\hline Hemoglobin & 0.993 & $0.985-1.002$ & 0.113 \\
\hline \multicolumn{4}{|l|}{ PSA baseline level } \\
\hline$\leq 65 \mathrm{ng} / \mathrm{ml}$ & 1.000 & & \\
\hline$>65 \mathrm{ng} / \mathrm{ml}$ & 2.036 & $1.411-2.939$ & $<0.001^{*}$ \\
\hline \multicolumn{4}{|l|}{ nPSA } \\
\hline$\leq 0.2 \mathrm{ng} / \mathrm{ml}$ & 1.000 & & \\
\hline$>0.2 \mathrm{ng} / \mathrm{ml}$ & 6.172 & $3.944-9.658$ & $<0.001^{*}$ \\
\hline \multicolumn{4}{|l|}{ TTN } \\
\hline$<6$ months & 1.000 & & \\
\hline$\geq 6$ months & 0.111 & $0.072-0.170$ & $<0.001^{*}$ \\
\hline
\end{tabular}

Table 3. Multivariate analysis for progression to metastatic castration resistant prostate cancer.

\begin{tabular}{llll}
\hline & HR & $95 \%$ CI & P Value \\
\hline $\begin{array}{l}\text { Gleason grade group } \\
\text { Grade group1 } 6 \text { 6 points }\end{array}$ & 1.000 & & \\
Grade group2 3+4 points & 1.512 & $0.556-4.108$ & 0.418 \\
Grade group3 4+3 points & 3.169 & $1.403-7.160$ & $0.006^{*}$ \\
Grade group4 8 points & 4.335 & $1.901-9.884$ & $<0.001^{*}$ \\
Grade group5 $\geq 9$ points & 5.159 & $2.312-11.512$ & $<0.001^{*}$ \\
P for trend <0.001* & & & \\
Limb bone metastasis & & & \\
No & 1.000 & & \\
Yes & 1.164 & $0.787-1.724$ & 0.447 \\
Visceral metastasis & & & \\
No & 1.000 & & \\
Yes & 0.737 & $0.419-1.297$ & 0.290 \\
Alkaline phosphatase & 1.000 & $0.999-1.001$ & 0.931 \\
PSA baseline level & & & \\
$\leq 65 \mathrm{ng} / \mathrm{ml}$ & 1.000 & & \\
$>65 \mathrm{ng} / \mathrm{ml}$ & 1.141 & $0.776-1.677$ & 0.501 \\
nPSA & & & \\
$\leq 0.2 \mathrm{ng} / \mathrm{ml}$ & 1.000 & & \\
$>0.2 \mathrm{ng} / \mathrm{ml}$ & 2.665 & $1.495-4.750$ & $0.001^{*}$ \\
TTN & & & \\
$<6$ months & 1.000 & & \\
$\geq 6$ months & 0.262 & $0.161-0.426$ & $<0.001^{*}$ \\
\hline$*$ P $<0.05$ & & &
\end{tabular}

\section{Discussion}

The Gleason scoring system is a well-established predictor for staging, progression and prognosis in PCa [14]. Yang et al. [15] reported that Gleason score was associated with survival of PCa patients with bone metastasis. The overall survival of patients with Gleason score $\leq 7$ was significantly longer than in patients with score $>7$. Yigitbasi et al.[16] explored the 
survival time of patients with MPCa who underwent ADT and found that median survival time was 33, 19 and 13 months in patients with Gleason score of 2-4, 5-7 and 8-10, respectively. In this study, we found that patients with $\mathrm{mPCa}$ had easy progression to CRPC if they were classified into a high Gleason grade group. We demonstrated that patients in Gleason grade groups 3, 4 and 5 showed a 3.169-, 4.335- and 5.159-fold higher risk, respectively, of progression to CRPC when compared with those in group 1. The Gleason grade group is one of the risk factors for progression to CRPC in patients with $\mathrm{mPCa}$.

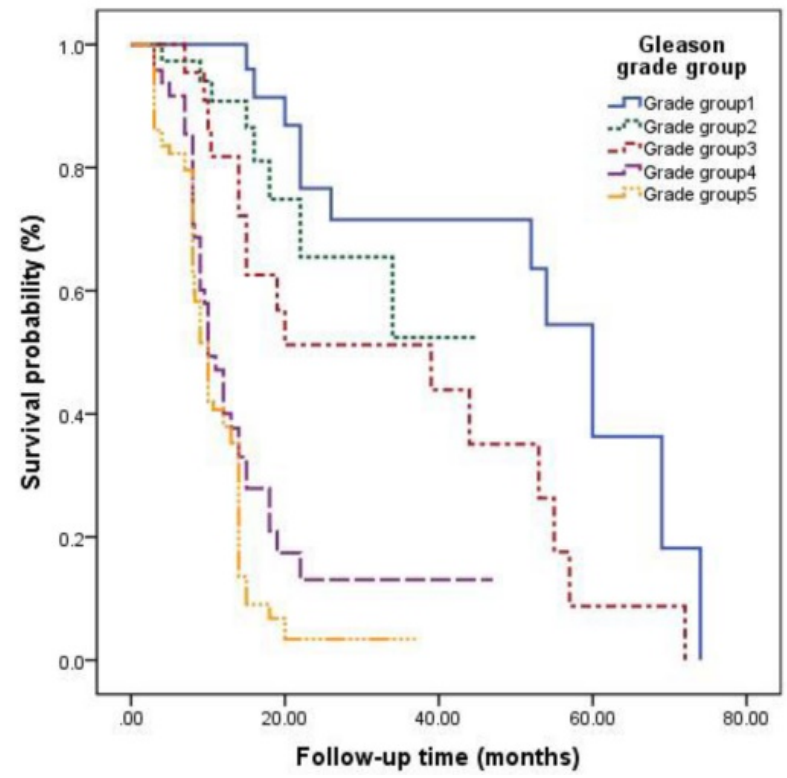

Figure 2. Kaplan-Meier analysis for progression-free survival in patients with advanced prostate cancer underwent androgen deprivation therapy stratified by Gleason grade group.

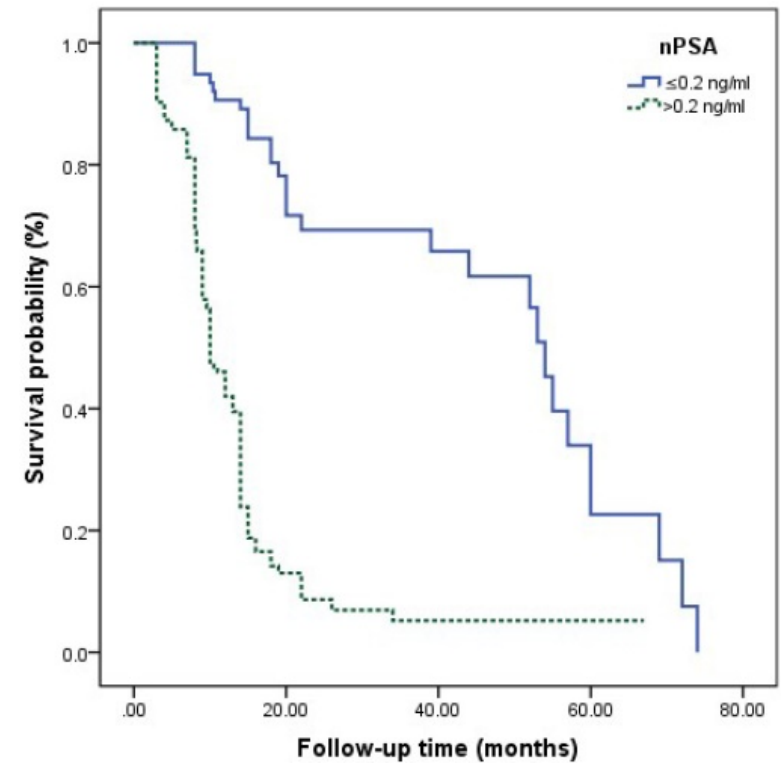

Figure 3. Kaplan-Meier analysis for progression-free survival in patients with advanced prostate cancer underwent androgen deprivation therapy stratified by nadir PSA.

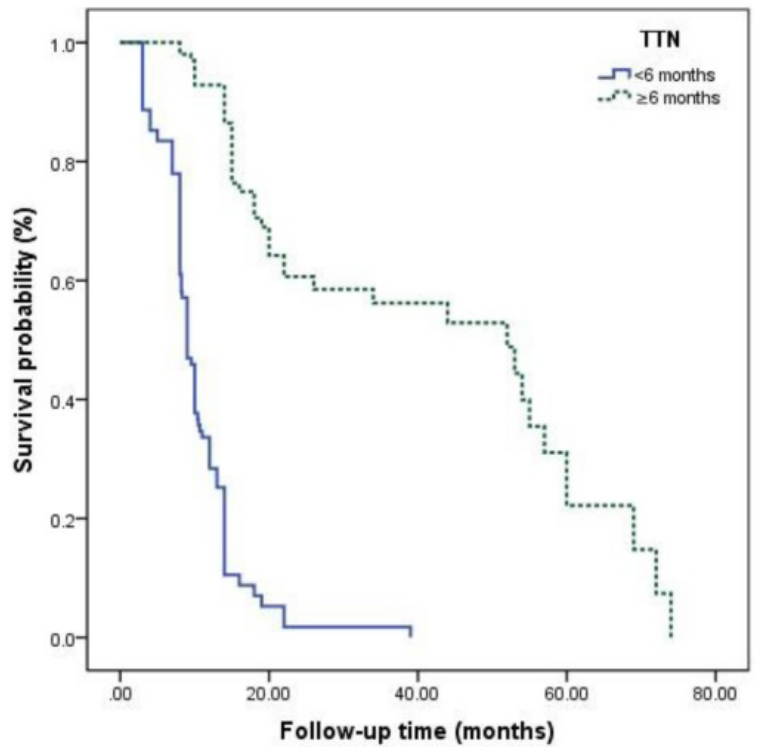

Figure 4. Kaplan-Meier analysis for progression-free survival in patients with advanced prostate cancer underwent androgen deprivation therapy stratified by time to nadir PSA.

For PCa, PSA is a widely used serological index in diagnosis, evaluation of therapeutic effect and prediction of prognosis [17-19]. Nayyar et al.[11] reported that higher PSA baseline level was associated with poorer therapeutic effect of ADT and shorter time to progression to CRCP. However, Yamamoto et al. [20] demonstrated that patients with PSA baseline level $<10 \mathrm{ng} / \mathrm{ml}$ had poorer therapeutic effect than those with $\geq 10 \mathrm{ng} / \mathrm{ml}$. Some previous studies also showed that PSA baseline level cannot work as a predictor for prognosis, which was supported by the present study [21, 22]. Recently, it was shown that nPSA and TTN seem to have better efficacy for prediction of prognosis than PSA baseline level has. Choueiri et al. [9] reported that TTN $<6$ months and nPSA $>0.2 \mathrm{ng} / \mathrm{ml}$ predicted shorter overall survival in patients who had hormonesensitive mPCa treated with ADT. A retrospective study about ADT for PCa or mPCa by Ji et al. [21] indicated that TTN $\leq 9$ months and nPSA $<0.03 \mathrm{ng} / \mathrm{ml}$ were significantly connected with an increased risk of progression to CRPC. Kuo et al. [23] reported a significant relationship between a longer time to PSA rise during the first off-treatment interval and a longer time to CRPC progression in patients treated with ADT. Besides, nPSA and TTN seem to work as predictors for prognosis in chemotherapy for CRPC [23]. It is generally considered that a rapid decline of PSA indicates a higher proportion of PCa cell death and, therefore, higher survival [24]. However, this is not consistent with what mentioned above. The underlying mechanisms are still unclear. It is possible that a rapid decrease of PSA might reflect 
down-regulation of PSA expression of hormone-sensitive PCa cells, which are regulated by androgens via the androgen receptor pathway [25]. Another possibility is that rapid removal of hormone-sensitive $\mathrm{PCa}$ cells might induce an environment that is conducive to the growth of CRPC cells [25]. Besides, a high nPSA means that many cancer cells develop into castration-resistant cells and survive ADT. Thus, more attention must be paid to patients with higher nPSA and shorter TTN for early identification of progression to CRPC.

Distant metastasis has been found in $>80 \%$ of patients who died of $\mathrm{PCa}[2]$. PCa usually tends to spread to axial bone rather than limb bone or viscera [26]. Rigaud et al. [26] reported that patients with axial bone metastasis have better survival than those with limb bone or visceral metastases. In addition, several studies have revealed that the presence of greater bone metastasis before ADT initiation results in earlier progression to CRPC [27].

The present study had several limitations. First, this was a retrospective analysis performed at a single institution, which restricts the application and generalization of our findings. We did not evaluate other biomarkers of androgen receptors because of technological limitations. Second, the univariate analysis revealed that limb bone metastasis, visceral metastasis and level of alkaline phosphatase might predict the progression to CRPC in patients with $\mathrm{mPCa}$ treated with ADT. However, multivariate analysis demonstrated opposite conclusions compared with univariate analysis. Third, multivariate analysis is more reliable than univariate analysis when considering the interaction among confounding factors. For instance, when compared to the studies of Rigaud [26] and Howard [27], patients included in this study have higher Gleason grade group, which possibly reduces the effects of bone metastasis and visceral metastasis towards the results. Finally, bias resulting from small sample size, relatively short follow-up time, and patient imposed selection are usually inevitable. Therefore, further study should be conducted to validate these conclusions.

In conclusion, this study demonstrated that higher Gleason grade group, higher nPSA, and shorter TTN were associated with higher risk of progression to CRPC in patients with mPCa. Further studies are needed to confirm these conclusions.

\section{Clinical Practice Points}

Patients with CRPC have poor prognosis and unsatisfactory therapeutic effects. This study demonstrated that Gleason grade group, nPSA and TTN were risk factors for progression to CRPC.
Patients with higher Gleason grade group, higher nPSA and shorter TTN have shorter PFS and higher risk of progression to CRPC after ADT. Further studies are needed to confirm these conclusions.

\section{Acknowledgments}

This study was supported by Foundation of Fujian Provincial Department of Finance (Grant number: 2018B011), the Joint Foundation of Fujian Province for Science and Technology Innovative Research Project (Grant number: 2017Y9093), Natural Science Foundation of Fujian Province (Grant number: 2017J01197), and Startup Fund for scientific research, Fujian Medical University (Grant number: 2017XQ2041).

\section{Authors' contributions}

Conception/Design: Ning $\mathrm{Xu}, \mathrm{Xue-Yi}$ Xue, Ting-Ting Lin; Provision of study material or patients: Yun-Zhi Lin, Ning Xu; Collection and/or assembly of data: Shao-Hao Chen, Shao-Zhan Chen, Xiao-Dong Li; Data analysis and interpretation: Ye-Hui Chen, XueYi Xue, Yu-Peng Wu; Manuscript writing: Ting-Ting Lin, Yu-Peng $\mathrm{Wu}$, Ye-Hui Chen, Shao-Zhan Chen; Final approval of manuscript: Qing-Shui Zheng, Yong Wei, Xue-Yi Xue.

\section{Availability of data and materials}

All data generated or analyzed during this study are included in this published article.

\section{Ethics approval and consent to participate}

This study was approved by the Ethics Committee of the First Affiliated Hospital of Fujian Medical University (Fuzhou, Fujian, China). All patients signed informed consent forms before they received ADT.

\section{Competing Interests}

The authors have declared that no competing interest exists.

\section{References}

1. Amato R, Stepankiw M, Gonzales P. A phase II trial of androgen deprivation therapy (ADT) plus chemotherapy as initial treatment for local failures or advanced prostate cancer. Cancer Chemother Pharmacol. 2013; 71(6): 1629-34.

2. Briganti A, Suardi N, Gallina A, Abdollah F, Novara G, Ficarra V, et al. Predicting the risk of bone metastasis in prostate cancer. Cancer Treat Rev. 2014; 40(1): 3-11.

3. Ciccarese C, Massari F, Jacovelli R, Fiorentino M, Montironi R, Di Nunno V, et al. Prostate cancer heterogeneity: Discovering novel molecular targets for therapy. Cancer Treat Rev. 2017; 54: 68-73.

4. Daniyal M, Siddiqui ZA, Akram M, Asif HM, Sultana S, Khan A. Epidemiology, etiology, diagnosis and treatment of prostate cancer. Asian Pac J Cancer Prev. 2014; 15(22): 9575-8.

5. Paner GP, Stadler WM, Hansel DE, Montironi R, Lin DW, Amin MB. Updates in the Eighth Edition of the Tumor-Node-Metastasis Staging Classification for Urologic Cancers. Eur Urol. 2018; 73(4): 560-569.

6. Winters-Stone KM, Moe E, Graff JN, Dieckmann NF, Stoyles S, Borsch C, et al. Falls and Frailty in Prostate Cancer Survivors: Current, Past, and Never Users of Androgen Deprivation Therapy. J Am Geriatr Soc. 2017; 65(7): 1414-1419. 
7. Merseburger AS, Alcaraz A, von Klot CA. Androgen deprivation therapy as backbone therapy in the management of prostate cancer. Onco Targets Ther. 2016; 9: 7263-7274.

8. Delahunt B, Egevad L, Srigley JR, Steigler A, Murray JD, Atkinson C, et al. Validation of International Society of Urological Pathology (ISUP) grading for prostatic adenocarcinoma in thin core biopsies using TROG 03.04 'RADAR' trial clinical data. Pathology. 2015; 47(6): 520-5

9. Choueiri TK, Xie W, D'Amico AV, Ross RW, Hu JC, Pomerantz M, et al. Time to prostate-specific antigen nadir independently predicts overall survival in patients who have metastatic hormone-sensitive prostate cancer treated with androgen-deprivation therapy. Cancer. 2009; 115(5): 981-7.

10. Divrik RT, Turkeri L, Sahin AF, Akdogan B, Ates F, Cal C, et al. Prediction of response to androgen deprivation therapy and castration resistance in primary metastatic prostate cancer. Urol Int. 2012; 88(1): 25-33.

11. Nayyar R, Sharma N, Gupta NP. Prognostic factors affecting progression and survival in metastatic prostate cancer. Urol Int. 2010; 84(2): 159-63.

12. Cornford P, Bellmunt J, Bolla M, Briers E, De Santis M, Gross T, et al. EAU-ESTRO-SIOG Guidelines on Prostate Cancer. Part II: Treatment of Relapsing, Metastatic, and Castration-Resistant Prostate Cancer. Eur Urol. 2017; 71(4): 630-642.

13. Eisenhauer EA, Therasse P, Bogaerts J, Schwartz LH, Sargent D, Ford R, et al. New response evaluation criteria in solid tumours: revised RECIST guideline (version 1.1). Eur J Cancer. 2009; 45(2): 228-47.

14. Pierorazio PM, Walsh PC, Partin AW, Epstein JI. Prognostic Gleason grade grouping: data based on the modified Gleason scoring system. BJU Int. 2013; 111(5): 753-60

15. Yang C, Qi GS, Rong RM, He J. Clinical features and treatment strategies for older prostate cancer patients with bone metastasis. Asian J Androl. 2013; 15(6): 759-63.

16. Yigitbasi O, Ozturk U, Goktug HN, Gucuk A, Bakirtas H. Prognostic factors in metastatic prostate cancer. Urol Oncol. 2011; 29(2): 162-5.

17. Filella X, Fernandez-Galan E, Fernandez Bonifacio R, Foj L. Emerging biomarkers in the diagnosis of prostate cancer. Pharmgenomics Pers Med. 2018; 11: 83-94.

18. Hendriks RJ, van Oort IM, Schalken JA. Blood-based and urinary prostate cancer biomarkers: a review and comparison of novel biomarkers for detection and treatment decisions. Prostate Cancer Prostatic Dis. 2017; 20(1): 12-19.

19. Grivas PD, Robins DM, Hussain M. Predicting response to hormonal therapy and survival in men with hormone sensitive metastatic prostate cancer. Crit Rev Oncol Hematol. 2013; 85(1): 82-93.

20. Yamamoto S, Ito T, Akiyama A, Aizawa T, Miki M, Tachibana M. M1 prostate cancer with a serum level of prostate-specific antigen less than $10 \mathrm{ng} / \mathrm{mL}$. Int J Urol. 2001; 8(7): 374-9.

21. Ji G, Song G, Huang C, He S, Zhou L. Rapidly decreasing level of prostate-specific antigen during initial androgen deprivation therapy is a risk factor for early progression to castration-resistant prostate cancer: A retrospective study. Medicine (Baltimore). 2017; 96(36): e7823.

22. Ji GJ, Huang C, Song G, Li XS, Song $Y$, Zhou LQ Predictive factor analysis of time to progression of castration-resistant prostate cancer after androgen deprivation therapy. Beijing Da Xue Xue Bao Yi Xue Ban. 2017; 49(4): 657-662.

23. Kuo KF, Hunter-Merrill R, Gulati R, Hall SP, Gambol TE, Higano CS, et al. Relationships between times to testosterone and prostate-specific antigen rises during the first off-treatment interval of intermittent androgen deprivation are prognostic for castration resistance in men with nonmetastatic prostate cancer. Clin Genitourin Cancer. 2015; 13(1): 10-6.

24. Facchini G, Caffo O, Ortega C, D'Aniello C, Di Napoli M, Cecere SC, et al. Very Early PSA Response to Abiraterone in mCRPC Patients: A Novel Prognostic Factor Predicting Overall Survival. Front Pharmacol. 2016; 7: 123.

25. Sasaki $T$, Onishi $T$, Hoshina A Nadir PSA level and time to PSA nadir following primary androgen deprivation therapy are the early survival predictors for prostate cancer patients with bone metastasis. Prostate Cancer Prostatic Dis. 2011; 14(3): 248-52.

26. Rigaud J, Tiguert R, Le Normand L, Karam G, Glemain P, Buzelin JM, et al. Prognostic value of bone scan in patients with metastatic prostate cancer treated initially with androgen deprivation therapy. J Urol. 2002; 168(4 Pt 1): $1423-6$.

27. Howard LE, De Hoedt AM, Aronson WJ, Kane CJ, Amling CL, Cooperberg $\mathrm{MR}$, et al. Do skeletal-related events predict overall survival in men with metastatic castration-resistant prostate cancer? Prostate Cancer Prostatic Dis. 2016; 19(4): 380-384 